Nloman 2018, 36(1), 41-50

Revista de Psicologia, Ciències de l'Educació i de l'Esport

ISSN: 1138-3194

Copyright (c) 2018

www.revistaaloma.net

\title{
Diferencias individuales, creencias de éxito y respuestas para tomar decisiones en diferentes tipologías del deportista
}

\author{
Juan González¹, Paula Rocamora², Francisco José Ortín ${ }^{2}$ \\ \& Enrique Javier Garcés de los Fayos ${ }^{2}$ \\ ${ }^{1}$ Universidad de Granada, ${ }^{2}$ Universidad de Murcia
}

Recibido: 19-01-2018

Aceptado: 08-04-2018

Diferencias individuales, creencias de éxito y respuestas para tomar decisiones en diferentes tipologías del deportista

Resumen. Señalar posibles indicadores psicológicos sobre cómo percibe su misma conducta, permite concretar las referencias sobre cómo orientar la misma de cara a ayudar al deportista a crecer a través del deporte que practica. En constante interactividad y bidireccionalidad (tanto positiva como negativa), las vivencias deportivas permiten la percepción de determinadas respuestas psicológicas, así como las características personales ejercen influencias determinantes para la práctica deportiva. Con el objetivo de describir y establecer relaciones entre algunas de estas diferencias individuales y contextuales, se realiza un estudio con una muestra aleatoria de jóvenes deportistas ( $N$ = 436). Utilizando cuestionarios de personalidad (BFQ), creencias sobre el éxito (POSQ) y toma de decisiones (CETD), se obtiene que la perseverancia es el indicador más característico de la muestra. Los deportistas federados difieren significativamente en dominancia, perseverancia y apertura a la experiencia, y los que practican deportes colectivos señalan significativamente mejor cooperación y apertura a la cultura que los que practican deportes individuales, que se orientan a ser más dominantes y estar más abiertos a nuevas experiencias. Además, los federados indican significativamente que están peor en ansiedad y que tienen un nivel más alto en competencia y compromiso para la toma de decisiones. Estos datos se reafirman cuando se establecen relaciones de correlación entre las variables estudiadas.

Palabras clave: motivación; jóvenes deportistas; decisiones; personalidad; deporte

Individual differences, beliefs about success and decision-making responses in different kinds of athletes

\begin{abstract}
Summary. By highlighting possible psychological indicators of how one perceives one's own behaviour, we can come to a more specific notion of how to orient athletes' behaviour toward personal growth via sport. Marked by constant interactive and bidirectional relationships (both positive and negative), experiences in sport reveal how certain psychological responses and personal characteristics can exert a decisive influence on performance. In order to describe and set out the relationships between some of these individual and contextual differences, a study was carried out on a random sample of young athletes $(N=436)$. Data were collected using a personality questionnaire (BFQ), measures of believes about success (POSQ) and a questionnaire on decision-making styles (CETD). The findings indicate that perseverance is the most common trait in the sample, that athletes who were members of athletic federations display significant differences with respect to dominance, perseverance and openness to experience, and that those who play team sports have significantly higher levels of cooperation and openness to culture than those who play individual sports, who are more likely to be dominant and open to new experiences. Additionally, members of federations display significantly higher levels of anxiety and of competitiveness and commitment to decision making. These data are reaffirmed by the correlations established among the variables studied.
\end{abstract}

Keywords: motivation; young athletes; decisions; personality; sport 


\section{Introducción}

La relación que los individuos establecen con el contexto en el que se desenvuelven, genera una constante construcción de su forma de ser, de sentir y de comportarse. Al mismo tiempo, desarrollarse para una persona significa combinar y generar consciencia sobre sus condiciones más estables (personalidad), a través de las vivencias que ofrecen experiencias de aprendizaje en contextos determinados (Allen \& Laborde, 2014; Cervone \& Pervin, 2015; García-Naviera \& Ruiz-Barquín, 2016; González \& Valadez, 2016; Kaiseler, Polman, \& Nicholls, 2012; MacAdams \& Pals, 2006; Rauthmann, Sherman, Nave, \& Funder, 2015).

De manera más concreta, cuando una persona crece y se representa a través del ámbito en el que se desenvuelve (el deporte en este caso), señala diferencias con respecto al desarrollo individual en cuanto a las características que le identifican, genera esquemas de pensamientos (modelos cognitivos a través de creencias para alcanzar sus propósitos) que orientan y vinculan respuestas psicológicas funcionales (confianza en sí mismo, automotivación, optimismo, recursos resilientes, compromiso, fortaleza mental,...), o disfuncionales (ansiedad, depresión, burnout, agresividad, obsesión...), y/o conductuales (de superación, adictivas, de relación o contacto social, de abandono, de adherencia,...).

Cada vez con más frecuencia, la psicología del deporte centra la atención hacia aquellas cualidades mentales que facilitan una información útil, funcional y con aplicabilidad a diferentes conceptualizaciones deportivas: rendimiento deportivo (Laborde, Lautenbach, Allen, Herbert, \& Achtzehn, 2014), bienestar psicofísico saludable (Friedman \& Kern, 2014), inicio y continuidad de la práctica deportiva (Chalabaev, Sarrazin, Fontayne, Boiché, \& Clément-Guillotin, 2013; Kerr, 2014), buscando ofrecer una correspondencia con las vivencias reales, de contenido y orientadas a la adaptación psicológica de una persona en el contexto deportivo en el que se desenvuelve.

Hallazgos en estudios con pequeñas muestras han encontrado pocas diferencias de personalidad entre deportistas de elite y los que practican deporte recreativo (Davis \& Mogk, 1994, Frazier \& Snyder, 1991; Gat \& McWhirter, 1998), sin embargo, estudios con grandes muestras han mostrado importantes diferencias que apuntan hacia que los atletas de elite son más extrovertidos y emocionalmente estables que los atletas de nivel recreativo (Egloff \& Gruhn, 1996; Williams \& Parkin, 1980). Más recientemente, algunos estudios señalan que los atletas que compiten en ámbito nacional o internacional han mostrado niveles más bajos de neuroticismo y mayores niveles de conciencia y agradabilidad que los atletas que compiten en clubes o competiciones regionales (Allen, Greenlees, \& Jones, 2011).

En el caso de jóvenes deportistas, el aprendizaje deportivo convive a través de constantes valoraciones subjetivas sobre el éxito o fracaso que van alcanzando (tabla 1), basándose en referencias objetivas relacionadas con el contexto (Biddle, Atkin, Cavill, \& Foster, 2011; Tsiotsou, 2012), en sus propios recursos o capacidades (Coulter, Mallett, Singer, \& Gucciardi, 2016; Kumar, 2016; Malinauskas, Dumciene, Mamkus, \& Venckunas, 2014), en sus respuestas psicológicas (González \& Valadez, 2016), o en su bienestar-malestar psicológico (Disabato, Goodman, Kashdan, Short, \& Jarden, 2016; Ryff, 2013).

Lo que nos diferencia nos identifica, y nos permite mostrar comportamientos distintos, específicos y contrastables en determinadas circunstancias de aparente regularidad (Caprara \& Cervone, 2000; González, Garcés de los Fayos, \& Ortega, 2014), donde el contexto deportivo intermedia en el ajuste-desajuste entre las creencias iniciales (disposición mental cognitiva) (Bilalic, McLeold, \& Gobet, 2007; Cavallera, Passerini, \& Pepe, 2013; Hansen, Larson, \& Dworkin, 2003), objetivos propuestos (expectativas) y la toma de las decisiones que antecede a las conductas llevadas a cabo para su consecución (respuesta cognitivo-emocional) (Allen \& Laborde, 2014; Allen, et al., 2011 Infante, Goñi, \& Villarroel, 2011; Sallis, Prochaska, \& Taylor, 2000).

El modelo Big Five (McCrae \& Costa, 1992) supone un intento de unificación entre los distintos puntos de vista existentes hasta el momento en cuanto a la descripción de la personalidad, y se caracteriza por una particular concepción de la ciencia (empirismo), una particular metodología (análisis factorial) y un particular modelo estructural (rasgos dimensionales), propugnando que cualquier tipo de personalidad puede ser conceptualizada a partir de cinco amplias dimensiones ortogonales (ver figura 1). Considerados como rasgos de temperamento, independientemente de la influencia del ambiente, aunque su expresión pueda estar moldeada por él (McCrae et al., 2000), aun manteniendo consistencia transituacional (diferentes situaciones), su base genética, estabilidad temporal y estructura (Costa \& McCrae, 1990), dando lugar a una psicología de la personalidad más madura y funcional (Hampson, 2012; John, Naumann, \& Soto, 2008; Ozer \& Benet-Martínez, 2006). Rhodes y Smith (2006) señalan en un profundo metaanálisis sobre personalidad y actividad física, que los rasgos de personalidad pueden predecir los resultados de los individuos (p. ej., la satisfacción o salud), díadas (p. ej., compromiso de relaciones), grupos (p. ej., la cohesión del equipo) y los contextos socio-deportivos donde se desenvuelven ( $p$. ej., conductas de dopaje).

\section{La búsqueda del éxito como medida del rendimiento y autovaloración del deportista}

Dentro de estas características individuales, la orientación que el individuo realiza en forma de creencias sobre los logros a alcanzar con la práctica que se realiza o emprende, han señalado la importancia de utilizar modelos motivacionales y atribucionales (Gaudreau \& Braaten, 2016; Vealey, 2002), ya que aportan información fundamental para entender el proceso y sus con- 


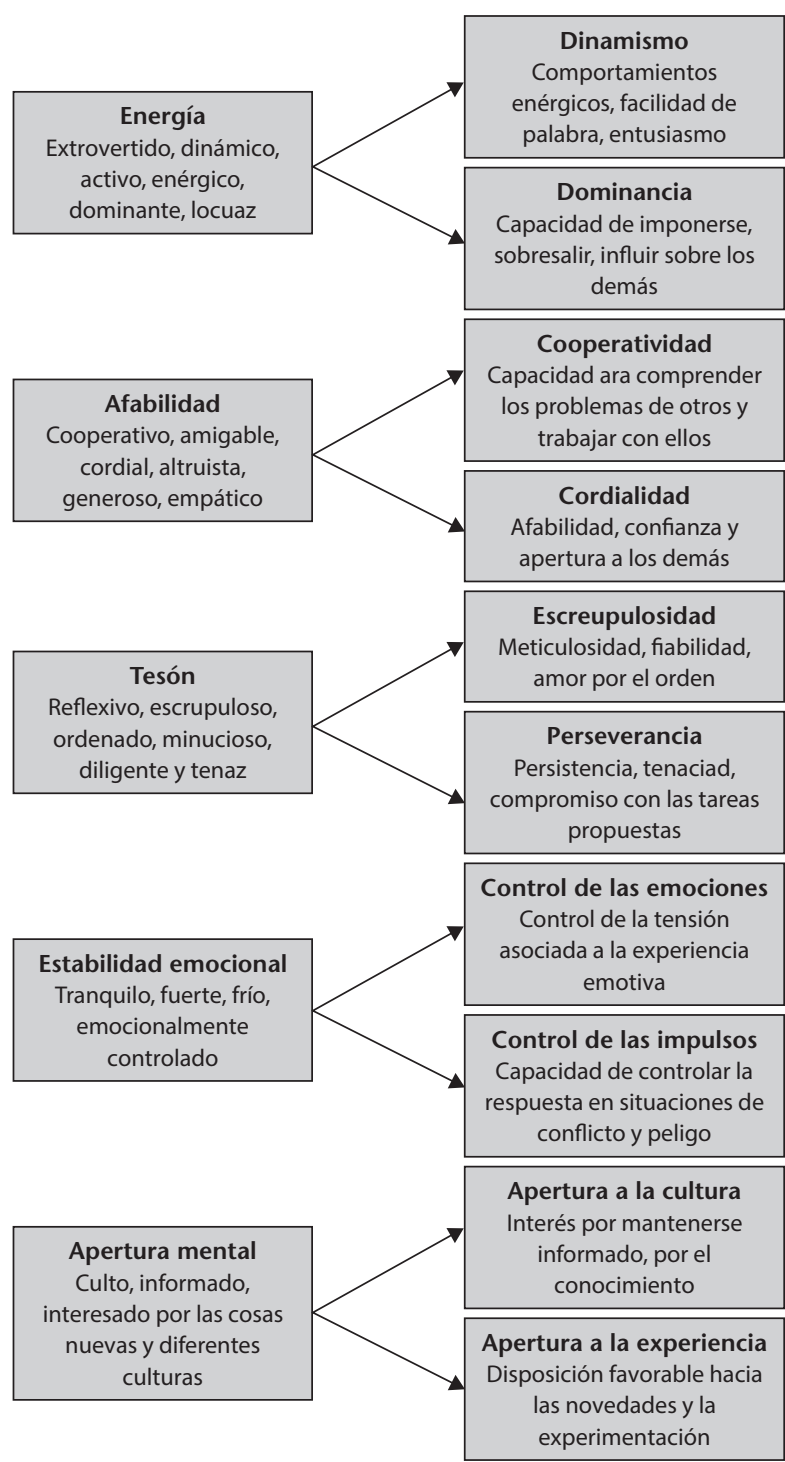

Figura 1. Estructura de dimensiones y subdimensiones de los Big Five.

secuencias. La teoría de las metas de logro (Nicholls, 1989), se centra en la importancia de la habilidad percibida en el proceso motivacional, distinguiendo entre orientación motivacional hacia el ego y hacia la tarea, haciendo referencia al concepto de habilidad percibida (Castillo, Balaguer, Duda, \& García-Merita, 2004; García-Mas \& Gimeno, 2008), atendiendo ade-

Tabla 1. Características de los deportistas con más éxito (Williams, 1993)

- Mantener el control y soportar la ansiedad.

- Mayor autoconfianza y autoinstrucciones más positivas.

- Mayores aspiraciones.

- Una imagen más frecuente sobre la naturaleza interna (imaginarse desde dentro del cuerpo, experimentando las mismas sensaciones que durante la ejecución física) que sobre la externa (verse a uno mismo como espectador).

- Mayor respuesta funcional ante las necesidades de rendimiento y exigencia deportiva en la preparación y en la competición.

- Mayor capacidad de bloquear o reducir la ansiedad antes y durante la competencia.

- Una mayor capacidad de enmendar sus errores.

- Disponen de los objetivos realistas por los que se considera un éxito o no éxito.

- Disponen de una capacidad motivacional autodeterminada, adecuada a los rasgos de personalidad de uno mismo.

- Coinciden con un liderazgo situacional de entrenadores, preparadores físicos, o incluso compañeros de equipo que les acompañan y/o dirigen en momentos concretos de la práctica deportiva. más a la influencia contextual como influyente en la citada elección motivacional (Duda, Cumming, \& Balaguer, 2005; Green \& Holeman, 2004; Usan, Salavera, Murillo, \& Álvarez-Medina, 2017). La tarea se torna aún más difícil ante la necesidad de considerar, en cada investigación, algunos factores situacionales (rivales, clima, clubes, entrenadores, padres, estado del campo de juego, influencia de árbitros, público, u otros significativos, las situaciones fortuitas, etc.), que interfieren en el rendimiento tanto o más que los factores hereditarios o aprendidos que "trae" el deportista (Woodman, Zourbanos, Hardy, Beattie, \& McQuillan, 2010).

\section{La toma de decisiones como elemento facilitador del rendimiento psicológico hacia el rendimiento deportivo}

Constantemente estamos tomando decisiones. En todas las circunstancias de la vida, nos encontramos con el proceso de elaboración de alternativas de pensamiento y actuación, ante las que debemos responder buscando la eficacia de las mismas. Al mismo tiempo, el riesgo que supone tomar una decisión dejando de lado otras alternativas, conlleva la implicación de mecanismos internos de autoconocimiento, confianza en sí mismos, autoestima, etc. que, a pesar de necesitar importantes procesos de consciencia, orientación sobre objetivos y autocontrol (Allen \& Laborde, 2014), supondrán bien seguridades y confirmaciones, bien dudas y temores sobre posibles errores que la decisión que hay que tomar cause en la estabilidad de uno mismo, o en el devenir de su rendimiento (Light, Harvey \& Mouchet, 2014).

La toma de decisiones, como “(...) proceso mental por el que la persona, tras una percepción del entorno, selecciona y planifica una respuesta idónea, compatible con el medio donde se encuentra, manifestada a través de una acción que se podrá comparar con el objetivo o patrón inicialmente propuesto" (Jiménez, 2007, p. 30), requiere previamente de un proceso de gestión mental de autoevaluación, que prepara el ajuste entre procesos cognitivos e individuales, los impulsos motivacionales y las circunstancias reales. El deportista se enfrenta a la decisión, en sus vertientes analítica (qué debo o puedo hacer), de elección (hago esto o lo otro) y de realización-control (lo estoy realizando o no), y experimenta una fase final de interpretación cognitivaemocional que potencia su repetición y continuidad, en caso de ser autoevaluada como positiva o generadora de oportunidades (tanto en intensidad como en reiteración de acciones) o de evitación y resistencia, en caso de ser autoevaluada como negativa o dificultosa.

La toma de decisiones, como proceso subjetivocognitivo (percepción de capacidad para sentirse competente por sí mismo y por entender lo que le llega de los demás), emocional (respuesta ansiosa y de incertidumbre o temor por repercutir la decisión que se tome) y de persistencia (compromiso con el aprendizaje técnico-táctico y continuidad por aprender) (Ruiz-Pérez, Palomo, García-Coll, Navia, \& Miñano, 2014), se ha asociado al rendimiento orientado hacia distraccio- 
nes en la atención de la tarea (Byrne, Silasi-Mansat, \& Worthy, 2015; Furley, Bertrams, Englert, \& Delphia, 2013), toma de elecciones finales (Raab, De Oliveira, \& Heinen, 2009; Ward, Suss, Eccles, Williams, \& Harris, 2011), o uso durante las acciones de juego (Johnson \& Raab, 2003; Laborde \& Raab, 2013 Raab, 2012), cuando se han querido explicar tanto los buenos como los malos rendimientos en el deporte.

\section{Objetivos del presente trabajo}

Se pretenden describir las orientaciones hacia el éxito, las características de personalidad y la capacidad para la toma de decisiones de una muestra de deportistas, para la diferenciación según la orientación deportiva (federada-no federada) y tipo de deporte (individualcolectivo), así como establecer relaciones sobre las variables ante cada tipología deportiva. Para ello, se asumirán las hipótesis que los indicadores de personalidad y perfiles de toma de decisiones señalarán diferencias significativas en el nivel y tipo de deporte, y que los indicadores de personalidad supondrán una influencia relevante en la gestión de la toma de decisiones en jóvenes deportistas, así como en la orientación motivacional hacia el éxito.

\section{Método}

\section{Participantes}

Con un diseño transversal, no aleatorio, descriptivo y relacional, el presente estudio se distribuye en población española, seleccionada de forma aleatoria, teniendo en cuenta el único criterio de inclusión de practicar actividad física de manera regular (3 veces por semana). La muestra (tabla 2) está compuesta por 436 deportistas, con edades comprendidas entre 13 y 23 años $(M=$ 16.83; $D T=2.37) ; 278$ de los participantes son hombres (63.76\%) y 158 mujeres (36.24\%), distribuidos y que cumplen la normalidad según el nivel de deporte (federado, 63.76\%-no federado, 36.29\%) y tipo de deporte (individual, 30.73\%-equipo, 69.27\%).

\section{Instrumentos}

Personalidad. Para el estudio de la personalidad se ha utilizado la adaptación al castellano del cuestionario Big Five (BFQ) (Caprara, Barbaranelli, Borgogni, \& Perugini, 1993. Adaptada a población española por Bermúdez, 1995. El test se compone de 132 ítems, distribuidos en 10 subdimensiones de 12 ítems cada una más una escala de distorsión, distribuida en escala
Likert de 1 (completamente falso) a 5 (completamente verdadero). Las dimensiones y subdimensiones que lo configuran son: Energía-Dinamismo, Dominancia, Afabilidad-Cooperación/Empatía, Cordialidad/Amabilidad, Tesón-Escrupulosidad, Perseverancia, Estabilidad Emocional-Control de Emociones, Control de Impulsos, Apertura Mental-Apertura a la Cultura y Apertura a la experiencia. El índice de fiabilidad para la muestra se señala en un $\alpha=.86$.

Toma de decisiones. Para el estudio de la toma de decisiones se utiliza el Cuestionario de Estilo Decisional en el Deporte (CETD) (Ruiz \& Graupera, 2005), que permite analizar el perfil decisional de los deportistas, incidiendo en la dimensión emocional de la toma de decisiones. Está compuesto por 30 ítems distribuidos en una escala Likert de 4 (totalmente en desacuerdototalmente de acuerdo). El cuestionario describe tres escalas de 10 ítems cada una, dos con orientación positiva: competencia decisional percibida (CDP) ("Me gusta tomar decisiones arriesgadas") y compromiso en el aprendizaje decisional (CAD) ("Me planteo las opciones que mi oponente puede tomar"), y una con orientación negativa ansiedad y agobio al decidir (AAD) ("Cuando fallo al decidir me gustaría desaparecer del campo de juego"). Se muestran de forma fiable en $\alpha=.83$.

Creencias hacia el éxito. Se ha utilizado el Cuestionario de Percepción de Éxito (POSQ) (Roberts \& Balagué, 1989, 1991), en su adaptación española por Cervelló, 1999). El POSQ es una escala de 12 ítems: 6 de Implicación en la tarea («"demuestro una clara mejoría personal", "trabajo duro") y 6 de Implicación en el yo («"soy claramente superior a los demás", «"mi actuación supera a mis rivales"). Los sujetos responden a la raíz inicial ("siento éxito en el deporte cuando..."), para posteriormente señalar las cuestiones formuladas en una escala tipo Likert con un rango de respuesta de 0 (totalmente en desacuerdo) a 100 (totalmente de acuerdo). La consistencia interna de las escalas ha mostrado un índice alfa de .86 para la escala de tarea y de .84 para la de ego.

\section{Procedimiento}

Siguiendo un mismo protocolo, se realizaron entrevistas iniciales con entrenadores o responsables (entornos profesionales) o deportistas (amateurs), con el fin de obtener la autorización necesaria para administrar la batería de cuestionarios; tras convocarlos grupalmente, completaban la batería de cuestionarios numerada para cada uno de los deportistas, y contestaban individualmente ante la presencia permanente de un evaluador para cualquier duda que surgiese. La participación de

Tabla 2. Distribución de los participantes de la muestra por rango de edad

\begin{tabular}{|c|c|c|c|c|c|}
\hline$N=436$ & & $>14-16$ años< & $>16-18$ años< & $>18-23$ años< & Total \\
\hline \multirow[t]{2}{*}{ NIVEL DEPORTE } & Individual & $38(28.35 \%)$ & $56(41.79 \%)$ & $40(29.85 \%)$ & 134 \\
\hline & Equipo & $92(30.46 \%)$ & $123(40.72 \%)$ & $87(28.80 \%)$ & 302 \\
\hline \multirow[t]{2}{*}{ TIPO DEPORTE } & Federado & $86(30.93 \%)$ & $118(42.44 \%)$ & $74(26.62 \%)$ & 278 \\
\hline & No federado & $56(35.44 \%)$ & $62(39.24 \%)$ & $40(25.32 \%)$ & 158 \\
\hline
\end{tabular}


todos fue voluntaria y aceptada mediante consentimiento informado que se adjuntaba a la batería de cuestionarios.

\section{Análisis de datos}

Mediante el análisis realizado con el programa estadístico SPSS 22.0, con la intención de mostrar la distribución muestral, se han obtenido medidas descriptivas (de frecuencias, tendencia central y dispersión) y de normalidad (K-S); para mostrar su fiabilidad se han realizado pruebas de consistencia interna (alfa de Cronbach); para la clasificación estadística en las agrupaciones establecidas se plantea un análisis descriptivo diferencial de variables psicológicas (análisis discriminantes), y para la relación entre las variables el análisis bidireccional de Pearson.

\section{Resultados}

En la tabla 3 se observa que las medias para las escalas de personalidad presentan valores entre control de impulsos (33.06) y perseverancia (44.75). Las medias de percepción de ego (26.49) son más altas que las de tarea (24.34), y en cuanto a las dimensiones en la toma de decisiones, se observa que las medias presentan valores entre el agobio al decidir (2.37) y el compromiso con la toma de decisiones (3.23).

En el contraste de las funciones discriminantes (tabla 4), se observa que existen diferencias significativas a favor de los practicantes de deportes de equipo en cooperación y apertura a la cultura, así como mayores indicadores de agobio en la toma de decisiones para los practicantes de deportes individuales, de orientación hacia el ego y de orientación hacia la tarea. Al mismo tiempo, se aprecian tendencias a la significación según si la modalidad deportiva es individual (dominancia) o de equipo (apertura a la experiencia). El análisis discriminante realizado entre deportistas individuales y de equipo es significativo, y la clasificación que establece se señala correctamente en un $78.43 \%$ en variables de personalidad, un $81.21 \%$ para las habilidades en la toma de decisiones, y un $75.52 \%$ en cuanto a la orientación motivacional.

De la misma forma, se aprecian diferencias significativamente discriminantes a favor de los deportistas federados en las cualidades de dominancia, perseverancia, capacidad percibida, compromiso en la toma de decisiones y orientación hacia el ego. En cuanto a los deportistas no federados, se diferencian significativamente en cordialidad, apertura a la experiencia, agobio a la hora de tomar decisiones y orientación hacia la tarea. El análisis discriminante es significativo, se clasifica correctamente, y se pueden afirmar las diferencias con una garantía del $75.08 \%$ en variables de personalidad, un $67.80 \%$ para las habilidades en la toma de decisiones, y un $72.03 \%$ en cuanto a la orientación motivacional.

Las relaciones de linealidad entre las variables indican importantes y significativos índices de correlación (tabla 5), y señalan entre los más importantes relaciones directas y moderadas entre competencia para la toma de decisiones con escrupulosidad, apertura a la experiencia, e inversas (aunque bajas) con cordialidad y cooperación.

La dimensión emocional de la ansiedad al decidir se relaciona de manera directa con la escrupulosidad y el control de emociones, y de manera inversa con el resto de características de personalidad, de manera que indicadores altos de personalidad protegen indicadores de ansiedad en el proceso de la toma de decisiones. El compromiso que se adquiere con la toma de decisiones se relaciona de manera directa con la mayoría de las variables de personalidad, así como con las creencias de éxito orientadas tanto hacia el ego como hacia la tarea.

También son cuantiosas las relaciones bidireccionales con alta y moderada significación, y destacan que conforme aumentan los niveles de dinamismo, cooperación, ansiedad y compromiso al decidir aumentan los indicadores de correlación de orientación hacia el ego, y conforme crecen la apertura a la experiencia y el compromiso decisional, crecen los de orientación hacia la tarea, el esfuerzo y los detalles de la mejora deportiva. Destacar, de la misma manera, las relaciones significativamente inversas entre un ostensible decrecimiento de la orientación hacia el ego, cuando existe una creciente apertura hacia la experiencia y cuando crece la percepción de competencia en la toma de decisiones. La orientación hacia la tarea decrece conforme existe un mayor dinamismo en la respuesta social de los deportistas.

\section{Discusión}

Con los propósitos de clasificar y establecer relaciones sobre variables psicológicas, según diferentes tipologías deportivas, se plantea un estudio diferencial y descriptivo en una muestra de deportistas españoles.

La muestra de deportistas elegida indica que los niveles más altos en sus puntuaciones son en perseverancia, lo que coincide con indicadores que transmiten otros artículos acerca de las características como el nivel deportivo (Weinberg, Butt, \& Culp, 2011), donde lo señalan como la característica que principalmente define a deportistas que alcanzan los mayores retos deportivos.

Se confirma la primera hipótesis planteada, mostrando diferencias significativas en personalidad, en cuanto a nivel de deporte, aunque no tanto sobre el tipo de deporte, asumiéndose de la misma forma por otros estudios (O'Sullivan, Zuckerman, \& Kraft, 1998). Ante el nivel de deporte, se observa que los federados se caracterizan por tener mayores niveles de dominancia, competencia y compromiso en la toma de decisiones, y mayor percepción hacia el ego. El grupo de los no federados se caracteriza principalmente por una mayor cordialidad, mayor apertura a la experiencia, mayor agobio en la toma de decisiones y una mayor orientación hacia la tarea. Aun no encontrando estudios completos que aporten similares resultados, con- 
Tabla 3. Estadísticos descriptivos de las dimensiones de personalidad, creencias de éxito y toma de decisiones

\begin{tabular}{|c|c|c|c|c|c|c|}
\hline$N=436$ & & Media & DT & Asimetría & Curtosis & K-S \\
\hline \multirow[t]{10}{*}{ PERSONALIDAD } & Dinamismo & 42.82 & 7.13 & .000 & -.420 & .38 \\
\hline & Dominancia & 38.81 & 6.22 & .168 & -.205 & .25 \\
\hline & Cooperación & 43.20 & 6.46 & -.348 & -.480 & .27 \\
\hline & $\underline{\text { Cordialidad }}$ & 40.40 & 5.67 & -.318 & -.008 & .32 \\
\hline & Escrupulosidad & 39.08 & 7.37 & -.031 & .191 & .41 \\
\hline & Perseverancia & 44.75 & 6.71 & .004 & -.184 & .24 \\
\hline & Control de emociones & 34.19 & 6.78 & -.306 & .737 & .17 \\
\hline & Control de impulsos & 33.06 & 7.15 & -.025 & -.040 & .21 \\
\hline & Apertura a la cultura & 37.68 & 5.95 & .179 & -.069 & .39 \\
\hline & Apertura a la experiencia & 42.07 & 6.28 & .206 & -.316 & .40 \\
\hline \multirow[t]{3}{*}{ TOMA DE DECISIONES } & $\mathrm{CAD}$ & 2.420 & 0.50 & .036 & -.170 & .44 \\
\hline & $\mathrm{AAD}$ & 2.378 & 0.66 & -.145 & -.752 & .34 \\
\hline & $\mathrm{CDP}$ & 3.234 & 0.46 & .155 & .134 & .26 \\
\hline \multirow[t]{2}{*}{ CREENCIAS DE ÉXITO } & Percepción EGO & 26.49 & 3.68 & -1.441 & 4.018 & .16 \\
\hline & Percepción TAREA & 24.34 & 3.65 & -.768 & 2.332 & .19 \\
\hline
\end{tabular}

CAD: competencia al decidir; AAD: agobio al decidir; CDP: compromiso con la toma de decisiones

Tabla 4. Diferencia de medias y coeficientes estandarizados (CE), según variables sociodemográficas de jóvenes deportistas

\begin{tabular}{|c|c|c|c|c|c|c|}
\hline \multirow[b]{2}{*}{ PERSONALIDAD } & \multicolumn{3}{|c|}{$n$ federado $=278 ; \mathrm{n}$ no federado $=158$} & \multicolumn{3}{|c|}{$n$ individual $=134 ; n$ equipo $=302$} \\
\hline & Lambda $\lambda$ & $t_{(217)}(p)$ & $\mathrm{CE}$ & Lambda $\lambda$ & $t_{(217)}(p)$ & CE \\
\hline Dinamismo & .803 & $-.778(.438)$ & -.156 & .800 & $.200(.842)$ & .028 \\
\hline$\underline{\text { Dominancia }}$ & .054 & $3.527(.001)$ & $.416^{* *}$ & .055 & $1.810(.060)$ & $.722^{\dagger}$ \\
\hline Cooperación & .901 & $-.546(.586)$ & .156 & .021 & $-2.164(.031)$ & $-.740^{*}$ \\
\hline Cordialidad & .037 & $-2.902(.004)$ & $-.409^{* *}$ & .703 & $-.764(.445)$ & -.100 \\
\hline Escrupulosidad & .811 & $1.529(.128)$ & .0322 & .607 & $1.248(.213)$ & .162 \\
\hline Perseverancia & .048 & $3.317(.001)$ & $.629^{* \star}$ & .002 & $-.598(.550)$ & -.198 \\
\hline Control de emociones & .708 & $1.317(.189)$ & .361 & .740 & $-.212(.832)$ & -.059 \\
\hline Control de impulsos & .801 & $-.535(.593)$ & -.080 & .804 & $.937(.349)$ & .282 \\
\hline Apertura a la cultura & .926 & $1.179(.240)$ & 0154 & .918 & $-2.003(.046)$ & $-.439^{*}$ \\
\hline Apertura a la experiencia & .015 & $-1.837(.048)$ & $-.575^{\star}$ & .015 & $-1.844(.056)$ & $-.553^{\dagger}$ \\
\hline \multicolumn{4}{|c|}{$\begin{array}{l}\text { Autovalor: .800; Wilks' Lambda: .176; correlación canónica: .408; X²: 38.593; sig .000; } \\
\text { reclasificación: } 75.08 \%\end{array}$} & \multicolumn{3}{|c|}{$\begin{array}{l}\text { Autovalor: .919; Wilks' Lambda: .117; correlación } \\
\text { canónica: .326; } X^{2}: 23.876 \text {; sig .008; reclasificación: 78.43\% }\end{array}$} \\
\hline TOMA DE DECISIONES & Lambda $\lambda$ & $t(217)$ & CE & Lambda $\lambda$ & $t(217)$ & $\mathrm{CE}$ \\
\hline $\mathrm{CAD}$ & .029 & $2.051(.041)$ & $.327^{*}$ & .602 & $.690(.491)$ & .276 \\
\hline $\mathrm{AAD}$ & .044 & $-4.171(.000)$ & $-.727^{\star \star}$ & .041 & $3.044(.003)$ & $.892^{\star *}$ \\
\hline $\mathrm{CDP}$ & .041 & $3.426(.001)$ & $.491^{\star *}$ & .577 & $-1.219(.224)$ & -.284 \\
\hline \multicolumn{4}{|c|}{$\begin{array}{l}\text { Autovalor: .734; Wilks' Lambda: .118; correlación canónica: .344; X²: 27.059; sig .000; } \\
\text { reclasificación: } 67.80 \%\end{array}$} & \multicolumn{3}{|c|}{$\begin{array}{l}\text { Autovalor: .849; Wilks' Lambda: .046; correlación } \\
\text { canónica: .216; } \mathrm{X}^{2}: 10.252 \text {; sig .017; reclasificación: } 81.21 \% \\
\end{array}$} \\
\hline CREENCIAS DE ÉXITO & Lambda $\lambda$ & $t(217)$ & CE & Lambda $\lambda$ & $t(217)$ & CE \\
\hline Percepción EGO & .015 & $6.180(.003)$ & $.841^{\star *}$ & .013 & $8.497(.004)$ & $-.497^{\star *}$ \\
\hline Percepción TAREA & .006 & $-3.696(.050)$ & $-.063^{*}$ & .045 & $22.665(.000)$ & $.562^{\star *}$ \\
\hline \multicolumn{4}{|c|}{$\begin{array}{l}\text { Autovalor: .985; Wilks' Lambda: .015; correlación canónica: .121; X²: 9.118; sig .000; } \\
\text { reclasificación: } 72.03 \%\end{array}$} & \multicolumn{3}{|c|}{$\begin{array}{l}\text { Autovalor: .963; Wilks' Lambda: .045; correlación } \\
\text { canónica: .188; } X^{2}: 22.340 ; \text { sig .000; reclasificación: } 75.52 \%\end{array}$} \\
\hline
\end{tabular}

Tabla 5. Relaciones de causalidad en la muestra de jóvenes deportistas, en cuanto a su personalidad, creencias hacia el éxito y capacidad para la toma de decisiones en jóvenes deportistas

\begin{tabular}{|c|c|c|c|c|c|c|c|c|c|c|c|c|c|c|c|}
\hline$N=436$ & 1 & 2 & 3 & 4 & 5 & 6 & 7 & 8 & 9 & 10 & 11 & 12 & 13 & 14 & 15 \\
\hline Dinamismo & 1 & $.361^{* *}$ & $.426^{* *}$ & $.333^{* *}$ & .023 & $.445^{* \star}$ & -.022 & $-.090^{*}$ & $.287^{* *}$ & $.518^{* *}$ & -.064 & $-.405^{* t}$ & $.307^{* *}$ & $.315^{* *}$ & $-.156^{* *}$ \\
\hline Dominancia & & 1 & $.114^{* *}$ & .077 & $.138^{* *}$ & $.418^{* *}$ & -.042 & $-.222^{* *}$ & $.145^{* *}$ & $.239^{* *}$ & $.131^{* *}$ & $-.151^{* *}$ & $.178^{* *}$ & $.198^{* *}$ & $.266^{* *}$ \\
\hline Cooperación & & & 1 & $.528^{* *}$ & $.144^{* *}$ & $.439^{* *}$ & .001 & $.082^{*}$ & $.196^{* *}$ & $.435^{* *}$ & $-.160^{* *}$ & $-.201^{* \star}$ & $.334^{* *}$ & $-.393^{* *}$ & $.175^{\star *}$ \\
\hline Cordialidad & & & & 1 & $.083^{*}$ & $.212^{* *}$ & .060 & $.221^{\star \star}$ & $.139^{* *}$ & $.360^{* *}$ & $-.174^{* *}$ & $-.430^{* *}$ & $.114^{* *}$ & $-.179^{* *}$ & .048 \\
\hline Escrupulosidad & & & & & 1 & $.302^{* *}$ & -.072 & .057 & $.164^{* *}$ & $.128^{* *}$ & $-.31^{\star}$ & $.584^{*}$ & $.117^{* \star}$ & .072 & -.011 \\
\hline Perseverancia & & & & & & 1 & -.005 & $-.088^{\star}$ & $.228^{* *}$ & $.420^{* *}$ & -.075 & $-.583^{* *}$ & $.397^{* *}$ & $.370^{* *}$ & $.211^{* *}$ \\
\hline Control emociones & & & & & & & 1 & $.496^{* *}$ & $.105^{* *}$ & -.006 & $.109^{* *}$ & $.480^{* *}$ & -.011 & -.023 & $.386^{*}$ \\
\hline Control impulsos & & & & & & & & 1 & .051 & .023 & .061 & -.044 & -.001 & -.016 & $.128^{\star *}$ \\
\hline Apertura cultura & & & & & & & & & 1 & $.447^{* *}$ & $.50^{*}$ & $-.517^{* \star}$ & $.150^{* *}$ & $.105^{* *}$ & .008 \\
\hline Apertura experiencia & & & & & & & & & & 1 & -.066 & $-.653^{*+}$ & $.554^{* *}$ & $-.665^{* *}$ & $.509^{* *}$ \\
\hline CAD & & & & & & & & & & & 1 & .019 & $.148^{* *}$ & $-.301^{*}$ & .059 \\
\hline $\mathrm{AAD}$ & & & & & & & & & & & & 1 & $-.134^{* *}$ & $.429^{* *}$ & $.203^{*}$ \\
\hline $\mathrm{CDP}$ & & & & & & & & & & & & & 1 & $.323^{* *}$ & $.522^{* *}$ \\
\hline Orientación EGO & & & & & & & & & & & & & & 1 & $.672^{* *}$ \\
\hline Orientación TAREA & & & & & & & & & & & & & & & 1 \\
\hline
\end{tabular}


trasta principalmente la información que aportan otros autores (García-Naviera, Ruiz-Barquín, \& Pujals, 2011) acerca de la no existencia de diferencias en apertura mental. En cuanto a la toma de decisiones, la muestra de deportistas indica que se perciben competentes en la toma de decisiones como indicador principal, confirmando datos de estudios anteriores que indican una mayor ansiedad y menor competencia al decidir por parte de los deportistas más competitivos (García, Ruiz, \& Graupera, 2009; Light, Harvey, \& Mouchet, 2014; Zeelenberg, Nelissen, \& Pieters, 2008).

En cuanto a la clasificación entre deportes individuales y de equipo, el resultado es que los deportistas individuales intentan ser más dominantes, sienten más agobio al decidir y se orientan mucho más hacia la tarea, mientras que los que practican deportes de equipo son más cooperativos, tienen mayor apertura mental a la cultura y a las experiencias, y se orientan más hacia el ego. De esta manera, se contrasta parcialmente la literatura, que ofrece información sobre que los deportistas individuales ofrecen mayores capacidades de dominancia y los de equipo de cooperación (González, Garcés de los Fayos, \& Ortega, 2014; Rhodes \& Smith, 2006). Los datos obtenidos se relacionan además con las creencias de éxito, ya que una orientación hacia el ego se ha asociado principalmente a deportistas que eligen objetivos realmente retadores, difíciles, que demandan gran energía y tiempo para obtenerlos, y enfrentan sus deseos, y temores y algunos obstáculos que se presentan externamente hacia su mejora personal y competitiva frente a otros (Duda, Cumming, \& Balaguer, 2005; Usan, Salavera, Murillo, \& ÁlvarezMedina, 2017).

Cuando se establece una relación directa entre variables, es destacable que los deportistas, conforme se muestran más cordiales y cooperativos, aumentan el compromiso y disminuyen su percepción de competencia, aspecto importante que hay que tener en cuenta en deportes colectivos (Gaudreau \& Braaten, 2016), que confirma de esta manera la segunda hipótesis planteada. De la literatura relacionada con la personalidad en el ámbito deportivo, se desprende que las personas que practican actividad física o deporte, en cualquiera de sus modalidades o exigencia deportiva, disponen de características de personalidad estables y con niveles altos, y muestran carencias en conceptos de estabilidad emocional y de autocontrol, lo que puede corroborarse con estudios parecidos en poblaciones de deportistas (De Moor, Beem, Stubbe, Boomsma, \& De Geus, 2006; McKelvie, Lemieux, \& Scout, 2003; Szabo, 1992). En contraste con los resultados obtenidos en el presente trabajo, los indicadores de afabilidad son altos, y se muestran como una característica representativa de la personalidad de los deportistas, tal y como sí muestran otros estudios (Rhodes \& Smith; 2006; Ruiz-Barquín, 2008) que señalan menor afabilidad cuando se comparan deportistas con muestras de no deportistas.

Las numerosas relaciones entre las características de personalidad y los "indicadores psicológicos", per- miten confirmar las hipótesis propuestas inicialmente, así como continuar con la línea ya aportada sobre la creciente literatura científica, con la inclusión dentro de los modelos explicativos de las características de personalidad (Allen \& Laborde, 2014; Biddle, et al., 2014; González \& Valadez, 2016; Kaiseler, et al., 2012; Laborde, et al., 2014; MacAdams \& Pals, 2006; Malinauskas, et al., 2014; Rauthmann, et al., 2015).

\section{Conclusiones}

Fijar cómo las relaciones entre las características de personalidad y respuestas psicológicas, tales como la orientación motivacional o gestión cognitivo-emocional de la toma de decisiones, señalan una serie de elementos individuales que orientan la percepción de la construcción psicológica y social en contextos de actividad física y el deporte. Se puede extraer del presente trabajo que existe una importante influencia de indicadores psicológicos cuando se practica una actividad o tarea física, sobre la forma de actuar ante dicha actividad o tarea, de manera que aquellas personas que practican deportes, tanto de equipo como individuales, así como de manera federada (más competitiva) o no federada, se diferencian individualmente en el establecimiento de metas y retos, manteniendo un nivel de compromiso elevado, usando pensamientos y tomas de decisiones diferenciales, utilizan esfuerzos intensos, duraderos y perseverantes para dominar el problema, se sienten estimulados por aquellos obstáculos que exigen un mayor esfuerzo, perciben la actividad o tarea física como accesible, poco peligrosa, ilusionante y motivadora.

En ocasiones, las personas que practican deporte o actividad física que perciben claramente estos procesos, prestan menor atención a las "amenazas psicológicas" y a pensamientos molestos, transforman cognitivamente las situaciones difíciles en constructivas (Ruiz \& Arruza, 2005), fomentan el interés intrínseco y la implicación para superar la actividad, recuperan rápidamente la sensación de eficacia ante cualquier fracaso y atribuyen dicho fracaso a la insuficiencia de esfuerzo, enfocan las situaciones complejas con la seguridad de controlarlas, orientan de forma acertada su nivel de activación, muestran mayor satisfacción y, por lo tanto, logran mejores resultados deportivos.

Entre las principales limitaciones que el estudio ha mostrado, se señalan las dificultades para el acceso a la muestra, y la complejidad por la elección de las variables más relevantes como objeto de estudio, de tantas que influyen en el proceso de influencia sobre las respuestas psicológicas. En este sentido, y mostrada la evidencia de la utilidad de la información, los entornos deportivos deben mostrarse mucho más abiertos a la aportación científica, que enriquecerá sus procesos de trabajo, la gestión personal y adaptativa de las conductas deportivas, y al mismo tiempo, se permitirá una mayor apertura a la utilidad del entrenamiento psicológico de los recursos individuales para la práctica deportiva. 
Como elementos de continuidad en la presente línea de investigación, se hace necesario el diseño de trabajos de investigación que se dirijan a la mejora del conocimiento de la persona, del deportista, de su personalidad y de su contexto deportivo, como antesala del inicio de cualquier propuesta de trabajo encaminada a la mejora de resultados, o rendimientos relacionados con la actividad física (condición física) o deportiva (más competitiva). Tal orientación es la que va a permitir afinar e individualizar más, si cabe, las metodologías de trabajo, las recomendaciones e instrucciones, las formas de motivar o motivarse y los mensajes en este sentido que se utilizan, por parte de entrenadores y preparadores deportivos, para un mayor entendimiento de la influencia de las variables situacionales sobre las personales, para mejorar los procesos temporales de costes-beneficios en pos de una mejora deportiva.

En definitiva, ser capaces de contemplar la importancia que desde la variabilidad contextual y psicológica supone la práctica deportiva, teniendo en consideración las variables de personalidad (o diferencias individuales) en cada uno de los deportistas, su innegable influencia, donde cualquier interpretación actitudinal (incluso conductual), sería sesgada e inconclusa.

\section{Referencias}

Allen, M. S., \& Laborde, S. (2014). The role of personality in sport and physical activity. Current Directions in Psychological Science, 23(6), 460-465.

Allen, M. S., Greenlees, I., \& Jones, M. (2011). An investigation of the five-factor model of personality and coping behaviour in sport. Journal of Sports Sciences, 29(8), 841-850.

Bermúdez, J. (1995). Cuestionario "Big Five". Adaptación al castellano del cuestionario BFQ de Caprara, Barbaranelli, Borgogni, \& Perugini (1993). Madrid: Tea Ediciones.

Biddle, S. J. H., Atkin, A. J., Cavill, N., \& Foster, C. (2011). Correlates of physical activity in youth: A review of quantitative systematic reviews. International Review of Sport \& Exercise Psychology, 4, 25-49.

Bilalic M., McLeold, P., \& Gobet, F. (2007). Personality profiles of young chess players. Personality and Individual Differences, 42, 6, 901-910.

Byrne, K. A., Silasi-Mansat, C. D., \& Worthy, D. A. (2015). Who chokes under pressure? The Big Five personality traits and decision-making under pressure. Personality and Individual Differences, 74, 22-28.

Caprara, G. V., \& Cervone, D. (2000). Personality: Determinants, dynamics, and potentials. New York: Cambridge University Press.

Caprara, G. V., Barbaranelli, C., Borgogni, L., \& Perugini, M. (1993). The Big Five Questionnaire: A new Questionnaire for the measurement of the five factor model. Personality and Individual Differences, 15, 281288.

Castillo, I., Balaguer, I., Duda, J. L., \& García-Merita, M. L. (2004). Factores psicosociales asociados con la participación deportiva en la adolescencia. Revista Latinoamericana de Psicología, 36(3), 505-515.

Cavallera, G.M., Passerini, A., \& Pepe, A. (2013). Los rasgos de personalidad y el papel del género en los nadadores a nivel de ocio. Comportamiento Social y Personalidad, 41(4), 693-703.

Cervone, D., \& Pervin, L. A. (2015). Personality, Binder Ready Version: Theory and Research. John Wiley \& Sons. Chalabaev, A., Sarrazin, P., Fontayne, P., Boiché, J., \& Clément-Guillotin, C. (2013). The influence of sex stereotypes and gender roles on participation and performance in sport and exercise: Review and future directions. Psychology of Sport and Exercise, 14(2), 136-144.

Cervelló, E., Escartí, A. \& Balagué, G. (1999). Relaciones entre la orientación de meta disposicional y la satisfacción con los resultados deportivos, las creencias sobre las causas de éxito en el deporte y la diversión con la práctica deportiva. Revista de Psicología del Deporte, 8, 7-21.

Costa, P.T., Jr., \& McCrae, R. R. (1992). Revised NEO Personality Inventory (NEO-PI-R) and NEO Five-Factor Inventory (NEO- FFI) professional manual. Odessa, FL: Psychological Assessment Resources, Inc.

Coulter, T. J., Mallett, C. J., Singer, J. A., \& Gucciardi, D. F. (2016). Personality in sport and exercise psychology: Integrating a whole person perspective. International Journal of Sport and Exercise Psychology, 14(1), 23-41.

Davis, C., \& Mogk, J. P. (1994). Some personality correlates of interest and excellence in sport. International Journal of Sport Psychology, 25(2), 131-143.

De Moor, M. H., Beem, A. L., Stubbe, J. H., Boomsma, D. I., \& De Geus, E. J. (2006). Regular exercise, anxiety, depression and personality: A population-based study. Preventive Medicine, 42(4), 273-279.

Disabato, D. J., Goodman, F. R., Kashdan, T. B., Short, J. L., \& Jarden, A. (2016). Different types of wellbeing? A cross-cultural examination of hedonic and eudaimonic well-being. Psychological Assessment, 28(5), 471.

Duda, J. L., Cumming, J., \& Balaguer, I. (2005). Enhancing athletes' self-regulation, task involvement, and self-determination via psychological skills training. En D. Hackfort, J. L. Duda, \& R. Lider (eds), Handbook of Applied Sport Psychology Research. Morgantown, WV: Fitness Information Technology, 143-165.

Egloff, B., \& Gruhn, A. J. (1996). Personality and endurance sports. Personality and Individual Differences, 21(2), 223-229.

Frazier, J. A., \& Snyder, E. E. (1991). The underdog concept in sport. Sociology of Sport Journal, 8(4), 380-388.

Friedman, H. S., \& Kern, M. L. (2014). Personality, wellbeing, and health. Psychology, 65(1), 719.

Furley, P., Bertrams, A., Englert, C., \& Delphia, A. (2013). Ego depletion, attentional control, and decision making in sport. Psychology of Sport and Exercise, 14(6), 900-904.

García-Naviera, A., Ruiz-Barquín, R., \& Pujals, C. (2011). Diferencias en personalidad en función de la prácti- 
ca o no deportiva, nivel de competición y categoría por edad en jugadores de fútbol desde el modelo de Costa y McCrae. Revista de Psicología del Deporte, 20(1), 29-44.

García, V., Ruiz, L. M., \& Graupera, J. L. (2009). Perfiles decisionales de jugadores y jugadoras de voleibol de diferente nivel de pericia. Revista Internacional de Ciencias del Deporte, 14(5), 123-137.

García-Mas, A., \& Gimeno, F. (2008). La Teoría de Orientación de Metas y la enseñanza de la educación física: consideraciones prácticas. Revista Latinoamericana de Psicología, 40(3), 511-522.

García-Naviera, A., \& Ruiz-Barquín, R. (2016). Diferencias en personalidad en función de la práctica o no deportiva y categoría por edad en jugadores de fútbol de rendimiento desde el modelo de Costa y Mccrae. Revista Iberoamericana de Psicología del Ejercicio y el Deporte, 11(1), 23-29.

Gat, I., \& McWhirter, B. (1998). Personality characteristics of competitive and recreational cyclists. Journal of Sport Behavior, 21(4), 408.

González, J., Garcés de los Fayos, E., \& Ortega Toro, E. (2014). Avanzando en el camino de diferenciación psicológica del deportista. Ejemplos de diferencias en sexo y modalidad deportiva. Anuario de Psicología, 44(1).

González, J., \& Valadez, A. (2016). Personalidad y respuesta psicológica en deportistas. Representación temporal y adaptativa del proceso persona-deporte. Retos, 30, 211-215.

Gaudreau, P., \& Braaten, A. (2016). Achievement Goals and their Underlying Goal Motivation: Does it Matter Why Sport Participants Pursue their Goals? Psychologica Belgica, 56(3), 244-268.

Green, T. D., \& Holeman, S. (2004). Athletes'attributions for team performance: a theoretical test across sports and genders. Social Behavior and Personality, 32(2), 199-206.

Hampson, S. E. (2012). Personality processes: Mechanisms by which personality traits "get outside the skin". Annual Review of Psychology, 63, 315.

Hansen, D. M., Larson, R. W., \& Dworkin, J. B. (2003). What adolescents learn in organized youth activities: A survey of self reported developmental experiences. Journal of Research on Adolescence, 13(1), 25-55.

Infante, G., Goñi, A., \& Villarroel, J. D. (2011). Actividad física y autoconcepto, físico y general, a lo largo de la edad adulta. Revista de Psicología del Deporte, 20(2), 429-444.

Jiménez A. C. (2007). Análisis de las tomas de decisión en los deportes colectivos: Estrategias de las jugadoras aleros de baloncesto en posesión del balón. Sevilla: Wanceulen.

John, O. P., Naumann, L. P., \& Soto, C. J. (2008). Paradigm shift to the integrative big five trait taxonomy. Handbook of personality: Theory and Research, 3, 114158.

Johnson, J.G., \& Raab, M. (2003). Take the first: Optiongeneration and resulting choices. Organizational Behavior and Human Decision Processes, 91, 215-229.
Kaiseler, M., Polman, R. C., \& Nicholls, A. R. (2012). Effects of the Big Five personality dimensions on appraisal coping, and coping effectiveness in sport. European Journal of Sport Science, 12(1), 62-72.

Kerr, J. H. (2014). Motivation and emotion in sport: Reversal theory. Psychology Press.

Kumar, A. (2016). A study on mental toughness and sports competition anxiety for male and female basketball players. International Journal of Physical Education, Sports and Health, 3(2), 379-381.

Laborde, S., \& Raab, M. (2013). The tale of hearts and reason: the influence of mood on decision making. Journal of Sport \& Exercise Psychology, 35(4), 339-357.

Laborde, S., Lautenbach, F., Allen, M. S., Herbert, C., \& Achtzehn, S. (2014). The role of trait emotional intelligence in emotion regulation and performance under pressure. Personality and Individual Differences, 57, 43-47.

Light, R. L., Harvey, S., \& Mouchet, A. (2014). Improving 'at-action'decision-making in team sports through a holistic coaching approach. Sport, Education and Society, 19(3), 258-275.

Malinauskas, R., Dumciene, A., Mamkus, G., \& Venckunas, T. (2014). Personality traits and exercise capacity in male athletes and non-athletes. Perceptual and Motor Skills, 118(1), 145-161.

McCrae, R. R., \& Costa Jr, P. T. (1997). Personality trait structure as a human universal. American Psychologist, 52(5), 509.

McCrae, R. R., Costa Jr, P. T., Ostendorf, F., Angleitner, A., H ebí ková, M., Avia, M. D., ... \& Saunders, P. R. (2000). Nature over nurture: Temperament, personality, and life span development. Journal of Personality and Social Psychology, 78(1), 173-186.

McKelvie, S. J., Lemieux, P., \& Stout, D. (2003). Extraversion and neuroticism in contact athletes, noncontact athletes, and non-athletes: A research note. Athletic Insight, 5.

Nicholls, J. G. (1989). The Competitive Ethos and Democratic Education. Cambridge, MA: Harvard University Press.

O'Sullivan, D., Zuckerman, M., \& Kraft, M. (1998). Personality characteristics of male and female participants in team sports. Personality and Individual Differences, 25, 119-128.

Ozer, D. J., \& Benet-Martínez, V. (2005). Personality and the prediction of consequential outcomes. Annual Review of Psychology, 57, 401-421.

Raab, M. (2012). Simple heuristics in sports. International Review of Sport and Exercise Psychology, 5, 104-120.

Raab, M., De Oliveira, R.F., \& Heinen, T. (2009). How do people perceive and generate options? Progress in Brain Research, 174, 49-59.

Rauthmann, J. F., Sherman, R. A., Nave, C. S., \& Funder, D. C. (2015). Personality-driven situation experience, contact, and construal: How people's personality traits predict characteristics of their situations in daily life. Journal of Research in Personality, 55, 98-111.

Rhodes, R. E., \& Smith, N. (2006). Personality correlates of physical activity: a review and meta-análisis. British Journal of Sports Medicine, 40(12), 958-965. 
Roberts, G.C. \& Balagué, G. (1989). The development of a social-cognitive scale of motivation. Paper presented at the Seventh World Congress of Sport Psychology, Singapore.

Roberts, G.C. \& Balagué, G. (1991). The development and validation of the Perception of Success Questionnaire. Paper presented at the FEPSAC Congress, Cologne, Germany.

Ruiz, L. M., \& Graupera, J. L. (2005). Dimensión subjetiva de la toma de decisiones en el deporte: desarrollo y validación del cuestionario CETD de estilo de decisión en el deporte. Motricidad. European Journal of Human Movement, 14, 95-107.

Ruiz, L. M., \& Arruza, J. A. (2005). El proceso de toma de decisiones en el deporte. Clave de la eficiencia y el rendimiento óptimo. Madrid: Paidós.

Ruiz-Barquín, R. (2008). Aportaciones del análisis subdimensional del cuestionario de personalidad $\mathrm{BFQ}$ para la predicción del rendimiento en judokas jóvenes de competición. Cuadernos de Psicología del Deporte, 8(1), 5-29.

Ruiz-Pérez, L. M., Palomo, M., García-Coll, V., Navia, J. A., \& Miñano, F. J. (2014). Self-perception of decision making competence in Spanish football players. Acta Gymnica, 44(2), 77-83.

Ryff, C. D. (2013). Psychological well-being revisited: Advances in the science and practice of eudaimonia. Psychotherapy and Psychosomatics, 83(1), 10-28.

Sallis J., Prochaska J., \& Taylor W. (2000). A review of correlates of physical activity of children and adolescents. Medicine and Science in Sports and Exercise, 32, 963-975.

Szabo, S. (1992). Habitual participation in exercise and personality. Perceptual and Motor Skills, 74, 978.
Tsiotsou, R. (2012). Developing a scale for measuring the personality of sport teams. Journal of Services Marketing, 26(4), 238-252.

Usan, P., Salavera, C., Murillo, V., y Álvarez-Medina, J. (2017). Creencias y percepciones del éxito en futbolistas adolescentes. Diferencias entre categorías deportivas y posición clasificatoria. Retos, 31, 207-211.

Vealey, R. (2002). Personality and sport behavior. In Vealey, R., Horn, T. (Ed), Advances in sport psychology (2nd ed.) (pp. 43-74). Champaign, IL, US: Human Kinetics.

Ward, P., Suss, J., Eccles, D. W., Williams, A. M., \& Harris, K. R. (2011). Skill-based differences in option generation in a complex task: A verbal protocol analysis. Cognitive Processing, 12, 289-300.

Weinberg, R., Butt, J., \& Culp, B. (2011). Coaches' views of mental toughness and how it is built. International Journal of Sport and Exercise Psychology, 9(2), 156-172.

Williams, J. M. E. (1993). Applied sport psychology: Personal growth to peak performance. Mayfield Publishing Co.

Williams, L. R., \& Parkin, W. A. (1980). Personality factor profiles of three hockey groups. International Journal of Sport Psychology, 11(2), 113-120.

Woodman, T., Zourbanos, N., Hardy, L., Beattie, S., \& McQuillan, A. (2010). Do performance strategies moderate the relationship between personality and training behaviors? An exploratory study. Journal of Applied Sport Psychology, 22(2), 183-197.

Zeelenberg, M., Nelissen, R. M. A., \& Pieters, R. (2008). Emotion, motivation, decision making: A feeling-isfor-doing approach. En H. Plessner, C. Betsch, y T. Betsch (Eds.), Intuition in judgment and decision making (pp. 173-190). Mahwah, NJ: Erlbaum. 